\title{
Introduction to Fifth Special Issue on Electroporation-Based Technologies and Treatments
}

\author{
Damijan Miklavčič ${ }^{1}$ Lluis M. Mir² ${ }^{2}$ P. Thomas Vernier $^{3}$
}

Published online: 25 August 2015

(C) Springer Science+Business Media New York 2015

This special issue of the Journal of Membrane Biology contains reports on recent developments in the field of electroporation by participants in the International Workshop and Postgraduate Course on Electroporation-Based Technologies and Treatments held in November 2014 in Ljubljana. This was the eighth session of what is now an annual event, first organized in 2003. The 66 participantsstudents, young scientists, invited lecturers, faculty members, and special guests-came from 15 countries (Algeria, Belgium, China, Croatia, France, Germany, Ireland, Israel, Italy, Lithuania, Macedonia, Netherlands, Slovenia, Spain, and United States). In addition to lectures on the physics, electrical engineering, and biology of electroporation, this year's sessions included topical talks on electrochemical processes associated with pulsed electric field exposures, electroporation in food and biomass processing, systemic electrochemotherapy, and electrochemotherapy of colorectal liver metastases. One of the features of this week-long workshop course is the practical training offered each afternoon. Organized and led by associates of the Laboratory of Biocybernetics of the Faculty of Electrical Engineering at

P. Thomas Vernier pvernier@odu.edu

1 Department of Biomedical Engineering, Laboratory of Biocybernetics, Faculty of Electrical Engineering, University of Ljubljana, Ljubljana, Slovenia

2 Laboratory of Vectorology and Anticancer Therapeutics, Centre National de la Recherche Scientifique (CNRS), University Paris-Sud (University Paris-Saclay), Institut Gustave-Roussy, European Associated Laboratory on the Pulsed Electric Fields Applications in Biology and Medicine, Villejuif, France

3 Frank Reidy Research Center for Bioelectrics, Old Dominion University, Norfolk, VA, USA the University of Ljubljana, and by visiting researchers, these laboratory sessions provide a unique opportunity for hands-on experience guided by experts, with activities including gene electrotransfer, ratiometric fluorescence microscopy, microbial inactivation, electrical characterization of artificial membranes, treatment planning for electrochemotherapy, and molecular dynamics simulations. The school is now an established and unique platform for the acquisition of theoretical and practical knowledge of electroporation mechanisms and applications.

The workshop and postgraduate course were conducted within the scope and with the support of the European Associated Laboratory on Pulsed Electric Fields Applications in Biology and Medicine (LEA EBAM) and co-organized by COST TD1104 Action (http://www. electroporation.net/).

The peer-reviewed selection of articles in this issue provides a cross section of the ongoing electroporationrelated research being carried out by participants at the Ljubljana meeting. We are grateful to the contributors for their efforts in presenting their recent results, which will challenge readers, whether new or old in the field, to evaluate assumptions and consider new ideas, and we are especially grateful to our scientific colleagues who reviewed the manuscripts.

Finally, we acknowledge the indispensable support of the agencies, societies, and companies who sponsor the school, in particular, the Slovenian Research Agency, the Centre National de la Recherche Scientifique (CNRS), and the Bioelectrochemical Society, which have sponsored the school from its very beginning, and also IGEA (Italy), Mediline (Slovenia), Iskra Medical (Slovenia), BIA Separations (Slovenia), LTFE (Slovenia), and Betatech (France), who made it possible to increase student participation through reduced fees and lodging expenses. 
COST (European Cooperation in Science and Technology) is a pan-European intergovernmental framework. Its mission is to enable break-through scientific and technological developments leading to new concepts and products and thereby contribute to strengthening Europe's research and innovation capacities.

It allows researchers, engineers, and scholars to jointly develop their own ideas and take new initiatives across all fields of science and technology, while promoting multiand interdisciplinary approaches. COST aims at fostering a better integration of less research intensive countries to the knowledge hubs of the European Research Area. The COST Association, an International not-for-profit Association under Belgian Law, integrates all management, governing, and administrative functions necessary for the operation of the framework. The COST Association has currently 36 Member Countries.
More information about COST is available at www.cost. eu.
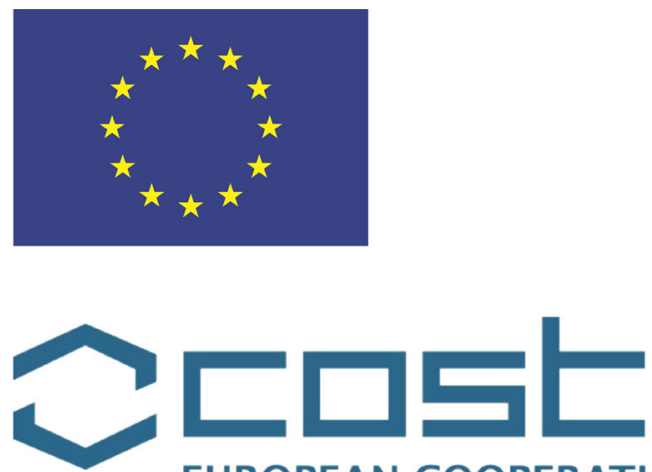

EUROPEAN COOPERATION IN SCIENCE AND TECHNOLOGY 